\title{
A New Procedure for the Fabrication of Custom Ocular Prosthesis - A Case Report
}
Ravi Shankar $\mathbf{Y}^{1}$
Srinivas $\mathbf{K}^{2}$

\author{
Manmeet Singh $^{3}$ \\ Anuj Wangoo ${ }^{4}$
}

\begin{abstract}
The eye is a vital organ and an important component of facial expression. Loss of an eye has a crippling effect on the appearance and psychology of the patient. Surgical removal of the eye is therefore normally followed by fabrication of an ocular prosthesis to improve esthetics. A cosmetically acceptable prosthesis is that which reproduces the natural color, contour, size and iris orientation. A sequence of steps for the construction of custom made ocular prosthesis is outlined in this case report using a modified impression technique.
\end{abstract}

Keywords: custom impression tray, ocular prosthesis, graphic grid.

\section{INTRODUCTION :}

The special sensory organs play significant role in our daily lives. The most tragic and most commonly occurring loss of one of these sensory organs is that of eye. The disfigurement associated with the loss of an eye can cause significant physical and psychological problems. Thus the replacement of the lost eye is necessary to promote physical and psychological healing for the patient and to improve social appearance ${ }^{1}$.

Ocular prosthesis are either ready-made or custommade $^{2}$. The need for an artificial eye can sometimes be fulfilled by stock prosthesis that comes in standard sizes, shapes, and colours. However, in majority of the enucleation cases custom ocular prosthesis is advantageous as there is improved adaptation to underlying tissues, increased mobility of the prosthesis, and better esthetics due to better match of the size and colour of the iris and sclera. Nevertheless, a custom prosthesis is more expensive than a stock prosthesis, and several steps and skills are required for its fabrication ${ }^{3}$.

The art of making artificial eyes has been known to man from the days of the early Egyptians. Simple inlaid eyes, consisting usually of white shell beads were found in Egypt dating back to 3000 B.C. ${ }^{4}$. Excavations of tombs have provided evidence of eye replacement by using precious stones, earthenware, copper, gold, enamelled bronze in the shrunken socket. Ambrose pare (1510-1590), a French man was the first to use both glass and porcelain eyes. By 1835 artificial eyes were being produced on a large scale in Germany, which continued as the centre of production. During the two world wars, the supply of glass eyes from Germany to United States was halted, and in 1943 the US Army and Navy both undertook research to find a substitute $^{4}$. Attention was concentrated on plastics, and the development of an acrylic eye resulted. Then onwards methyl methacrylate prosthesis became popular since they offered superior strength and the shape and size could be modified. Flexible material such as silicone ${ }^{5}$ became advantageous when the defect extends beyond the orbital area and encounters movable tissue beds. A more recent advancement is the use of orbital implants. Orbital implants are prosthetic devices of alloplastic material implanted into the orbital tissue beneath the conjunctiva, to fill the space of the orbit and facilitate in the process of fabrication of ocular prosthesis. Their use resulted in better retention and increased acceptance of the prosthesis.

The combined efforts of the ophthalmologist and the maxillofacial prosthodontist are needed to provide a satisfactory ocular prosthesis when trauma or disease causes the loss of an eye.

The following are the reasons for the removal of an $\mathrm{eye}^{6}$ :

- A blind painful eye

- A severely traumatized eye

- Eyes containing life threatening tumors (ex: melanoma)

- Presence of risk of sympathetic ophthalmia

- Poor cosmetic appearance of blind eye

\section{SURGICAL PROCEDURES IN THE REMOVAL OF} ANEYE ${ }^{6}$ :

- Evisceration- It involves the removal of the contents of the globe leaving in place the sclera and sometimes the cornea. The prosthesis best suited for the evisceration defect is the custom cover shell or the scleral cover shell prosthesis.

- Enucleation- Enucleation is the removal of the entire globe after the extra ocular muscles and the optic nerve have been transected. The prosthesis best suited for the defect is conventional or implant supported ocular prosthesis. Most surgeons will place an implant in 
tennons capsule to fill the orbital defect and support the muscles and eyelids. Use of an implant permitting attachment of the extra ocular muscles often enhances the mobility of the prosthesis.

- Exenteration- Exentration is the removal of the entire contents of the orbit (entire eye and surrounding structures). This procedure is usually performed due to some form of malignant disease.

Prosthetic rehabilitation of a patient with evisceration or enucleation will be greatly enhanced of an implant is placed in the orbit.

\section{CASE REPORT:}

A 12-year-old boy sustained a blow to his left eye that resulted in the loss of the eye. The surrounding bone provided a protective case for the globe contents and allowed the surgeon to preserve most of the muscle attachments and fatty tissue that cushions the eye.

\section{IMPRESSION TECHNIQUES}

Numerous impression techniques have been described in the literature. Most can be placed into one of several broad categories: Direct impression / external impression, Impression with stock ocular tray, Stock ocular tray modifications, Impression with custom ocular tray, Impression with stock ocular prosthesis, Ocular prosthesis modification, Wax scleral blank technique ${ }^{3}$.

\section{1) Direct impression / external impression technique :}

Alginate impression material is mixed and loaded into a disposable $60 \mathrm{ml}$ piston irrigation syringe and injected directly into the enucleated socket ${ }^{7,8}$ while the patient gazes directly forward at a fixed point at least 6 feet away. Additional material is applied to the external tissue and an impression is made using a rigid tray for reinforcement.

\section{2) Stock ocular tray impression technique :}

An impression is made of the ocular defect using a stock ocular tray ${ }^{9}$. Ophthalmic reversible hydrocolloid is mixed and loaded in the syringe, and sufficient material is ejected to fill the concavity of the tray. The tray is then oriented into the defect, sufficient material is injected to elevate the lid contours similar to the normal side. After sufficient time, the assembly is removed and the impression is examined for defects and voids.

\section{3) Stock ocular tray modification :}

Maloney placed 3 channels through the superior edge of his own set of customized stock trays to prevent air entrapment ${ }^{10}$.

\section{4) Impression with custom ocular tray :}

Miller suggested that a custom ocular tray is necessary in certain situations. Miller's method ${ }^{11}$ involves attaching a solid suction rod to the patient's existing prosthesis, conformer (or) wax shell and investing it in an alginate mold. After alginate sets, the prosthesis, conformer (or) wax is removed and replaced with clear acrylic resin. Perforations are made in the resulting tray and a tunnel is cut into the stem through which impression material can be delivered. An impression is made using injected alginate.

\section{5) Impression using stock ocular prosthesis:}

An Esthetic stock eye is selected. Its periphery and posterior aspect are reduced and lined with alginate and inserted for impression. It is then processed ${ }^{4}$.

\section{6) Ocular prosthesis modification:}

Smith described reline procedure for an existing prosthesis using Korecta wax no. $4^{12}$. Ow and Amrith used tissue conditioner as reline material after reducing the stock prosthesis. Viscogel is used and left for 24- 48 hours to create functional impression ${ }^{13}$.

\section{7) Wax scleral blank technique :}

Benson created a wax blank by adapting base plate wax around a steel ball ${ }^{14}$.

McKinstry made wax pattern based on his observation of site.

New impression technique: An impression of the socket was made with an ocular shaped impression tray by modifying the technique described by Miller $^{11}$. A prefabricated stock eye shell was selected. Impression of inner surface of the shell was made with alginate (Jeltrate, Densply ). After the setting of alginate, the stock eye shell was removed and replaced with clear acrylic resin (DPI RR cold cure) for the fabrication of custom tray. Perforations were made in the custom tray. The custom tray was attached to a $5 \mathrm{ml}$ disposable syringe (Dispovan). The custom tray was tried and overextensions were corrected.

Step 1 (impression of the anophthalmic socket): Border molding was done using polyvinyl siloxane putty impression material Aquasil - soft putty, Dentsply). Tray adhesive was applied to the borders of the tray. Putty material was adapted to the borders of the tray and inserted into the socket. Patient was instructed to perform eye movements while the putty sets. The tray was removed from the socket and the syringe was filled with light body PVS impression material (Aquasil, Light body- Dentsply.). The tray was reinserted and the light body material was injected into the socket. Patient was instructed to perform eye movements while the impression material sets. This allowed the impression material to flow over the underlying muscle bed and the anatomic details to be recorded accurately (Fig 1). 

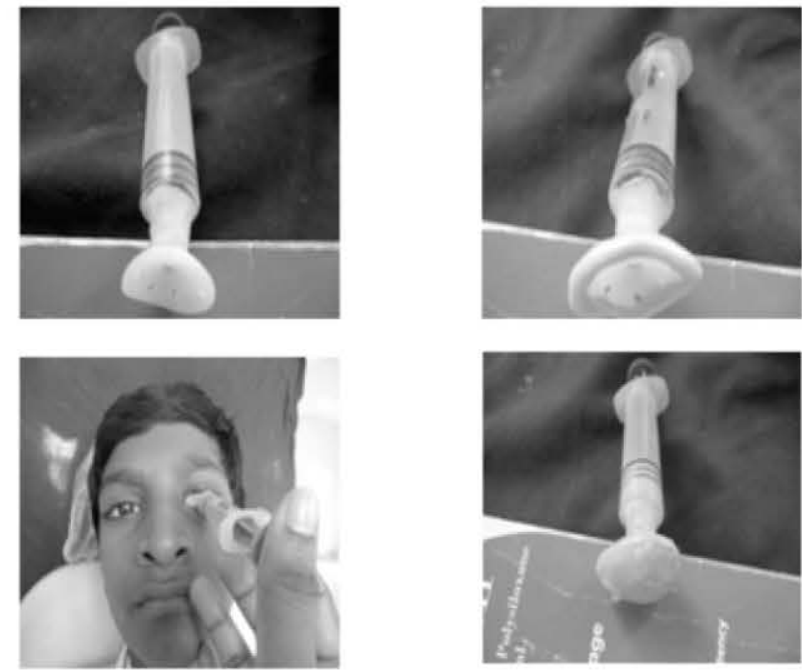

Fig 1: Impression of the defect

Step 2 (Impression of the outer surface): The syringe was cut out from the custom tray and a plastic stick was attached to the centre of the outer surface of the custom tray using cyanoacrylate. Light body material was added to the outer surface of the tray. The tray was reinserted into the socket and the patient was asked to close the eye slowly. With both eyes closed, it was made sure that the outer contours of the eye lids mimic those of the adjacent normal eye (Fig 2). This functional molding resulted in exact replication of the outer contours similar to that of the natural eye.

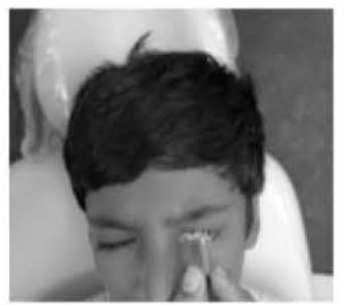

Fig 2: Recording the outer contour of the eye

Wax pattern fabrication: A two-piece dental stone mold of the impression was made. Softened wax was placed in the mold and the excess was forced out to make the wax pattern. Minimal alterations of the scleral wax pattern were done until satisfactory contours of the eyelids were achieved in open and closed position (Fig 3).
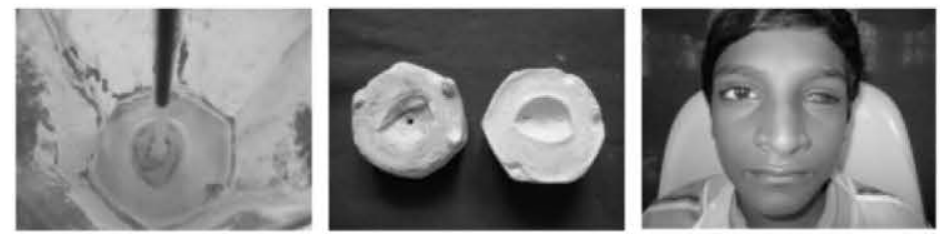

Fig 3: Fabrication and try-in of scleral wax pattern

Technique of iris disc placement: Various techniques like ocular locator $^{15}$, facial measurements ${ }^{15}$ and pupillometer 16 have been used in the past for iris disc placement.
In this case, a transparent graphic grid was used to position the iris disc. A vertical midline was marked passing through the forehead crease, glabella, tip of the nose and chin. The distance from the right eye medial canthus to the midline and left eye medial canthus to the midline was measured. This distance standardized the midline marking and was used to reposition the grid template each time during the try-in visit.

The patient was asked to gaze straight at an object kept 4 feet away. The operator then marked the vertical lines coinciding with the medial and distal extremities of the iris of the natural eye. Similarly the horizontal lines referring to the centre, inferior and superior limits of the iris were marked. The facial markings were transferred to the grid template by placing it on the patients face. These markings were transferred to the side of the defect. These markings were transferred to the sculptured wax pattern ${ }^{17}$. The iris disc was shade matched with the adjacent eye and cut out from a stock eye. Various techniques like paper iris disc technique18, black iris disc technique ${ }^{18}$, photographic iris disc technique ${ }^{19}$ have been used in the past for the fabrication of iris disc. In this case, shade matched iris disc was cut out from a stock eye as it is a simpler method. The iris disc was attached to the wax pattern (Fig 4).
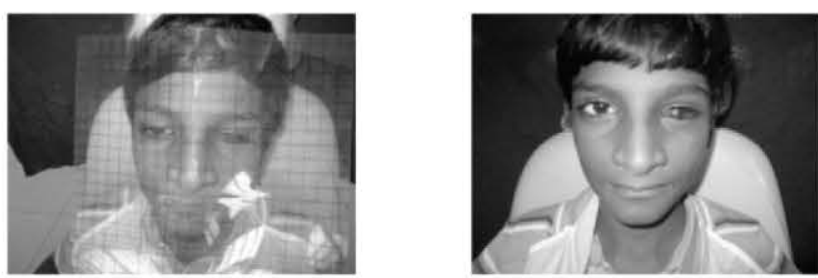

Fig 4: Iris disc placement using graphic grid

Investing, Dewaxing, Packing: The finished pattern was invested in a denture curing flask. A two part mold was constructed by the prototype ocular prosthesis by using gypsum. The flask was then placed in a dewaxed bath for 20 $\mathrm{min}$. The color of the sclera was selected using tooth color acrylic shade guide 15 .

A thin layer of clear acrylic was adapted on the outer surface of the dewaxed mold (Fig 5). Rayon thread fibrils were dispersed to simulate vasculature18. The selected shade of the sclera was matched with the heat cure resin (DPI) which was then packed in the two piece flask. The flask was kept for curing.

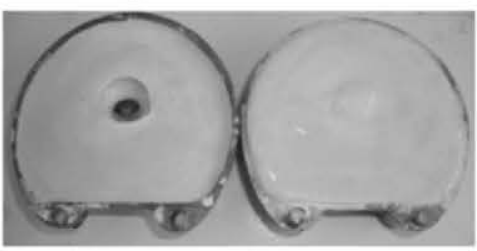

Fig 5: Dewaxed mold 
Placement of the prosthesis and follow up: After curing, the prosthesis was separated from the mold and was polished. The prosthesis was disinfected in a solution of $0.5 \%$ chlorhexidine for 15 minutes and rinsed in sterile saline solution. The custom ocular prosthesis was then inserted (Fig 6). The patient was instructed and trained on the aspects of insertion and easy removal of the prosthesis.
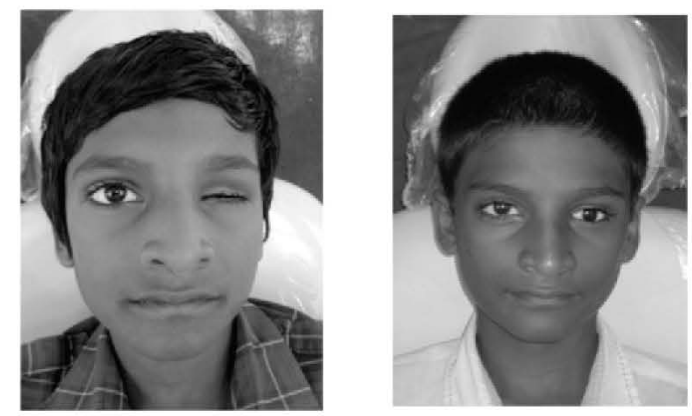

Fig 6: Final prosthesis polished and placed into the ocular defect.

\section{CONCLUSION}

A simplistic procedure for fabricating the custom ocular prosthesis has been suggested here. The modified functional impression technique followed in this case allowed the artificial eye to move in unison with the patient's natural eye without being dislodged by these movements. Due to intimate contact of the prosthesis with the tissue surface of the defect, movement of the prosthesis increased and enhanced the natural appearance of the prosthesis. The impression making on the dorsal aspect of the tray made sure that the outer contours of the eye lids mimic those of the adjacent normal eye This created a pleasing esthetic and functional result. The technique has provided good results from patient esthetics, acceptance, and satisfaction point of view.

\section{REFERENCES:}

1. Artopoulou I, Montgomery P, Wesley P, Lemon J. Digital imaging in the fabrication of ocular prostheses. The Journal of prosthetic dentistry2006;95(4):327-30.

2. Erypf S F. Comparative features of plastic and glass in artificial eye construction. Arch Ophthalmol 1953; 50: 737-8.

3. Mathews FM. The ocular impression: a review of the literature and presentation of an alternate technique. J P rosthodont 2000; 9: 210216.

4. Chalian VA. Maxillofacial prosthetics - Multidisciplinary practice. The Williams and Wilkins company 1972: 286-7.

5. Manvi Supriya. Prosthodontic rehabilitation of a patient with an orbital defect using a simplified approach. JIPS 2008; 8(2): 116-118.

6. Parr GR. Surgical considerations in the prosthetic treatment of ocular and orbital defects. JPD 1983; 49(3): 379-85.

7. Bartlett S. ocular prosthesis: a physiological system. JPD 1973; 29(4): 450-9.

8. Brown K. Fabrication of an ocular prosthesis. JPD 1970; 24(2): 225235.

9. Allen L. Modified impression method of artificial eye fitting. American Journal of Ophthalmology 1969; 67(2): 189-218.

10. Maloney B. development of impression fitting equipment: a new technique. J Am Soc Ocularists 1979; 9: 32-3.

11. Miller B. Custom ocular impression trays. J Facial Somato Prosthet 1996;2:109-13.

12. Smith R. relining an ocular prosthesis: A case report. J Prosthodont 1995; 4 : 160-3.

13. Ow R. Ocular prosthesis: use of a tissue conditioner material to modify a stock ocular prosthesis. JPD 1997; 78(2): 218-222.

14. Benson $P$. The fitting and fabrication of a custom resin artificial eye. JPD 1977; 38(5): 532-8.

15. Mc Arthur D.A.; Aids for positioning prosthetic eyes in orbital prosthesis. J Prosthet Dent 1977;37:192-3.

16. Roberts AC. An instrument to achieve pupil alignment in eye prosthesis. JPD 1969; 22: 487.

17. Sinha ND. Fabrication of custom ocular prosthesis using a graph grid. Pravara Med Rev 2009; 4(1): 21-24.

18. Thomas D. Taylor. Clinical Maxillofacial prosthetics. Pg 233-76.

19. Jain S. Prosthetic rehabilitation of ocular defect using digital photography: A case report. JIPS 2010; 10(3) : 190-193. 